\title{
Single port access laparoscopic myomectomy with X-Cone
}

\author{
Liliana Mereu • Stefano Angioni • Alessandro Pontis • \\ Giada Carri $\cdot$ Luca Mencaglia
}

Received: 8 November 2010 / Accepted: 15 December 2010 / Published online: 11 January 2011

(C) Springer-Verlag 2011

\begin{abstract}
Single-port access laparoscopy (SPAL) technique is developing in the gynecological field but few experiences on SPAL myomectomy have been reported. A case of an infertile nulliparous woman who has been observed to have an intramural-subserosal myoma of $4 \mathrm{~cm}$ and bilateral sactosalpinx is reported. The patient underwent a SPAL to remove the myoma and bilateral sactosalpinx. No intraoperative and postoperative complications occurred. Total operative time was $94 \mathrm{~min}$. We describe the technique with strategies to perform surgical treatment of the myoma with a single-access laparoscopic approach.
\end{abstract}

Keywords Myomectomy · Laparoendoscopic single-site surgery $\cdot$ Single-port access $\cdot$ Minimal access surgery

\section{Background}

Leiomyomas represent the most common pelvic tumors, affecting $25 \%$ to $45 \%$ of women of reproductive age and occurring three times more frequently in African American

L. Mereu $(\bowtie) \cdot$ A. Pontis $\cdot$ G. Carri $\cdot$ L. Mencaglia

Division of Gynecology, Centro Oncologico Fiorentino,

Via Ragionieri 101, Sesto Fiorentino,

Florence, Italy

e-mail: Liliana.mereu@lacittadellasalute.it

S. Angioni · A. Pontis

Division of Gynecology, Obstetrics and Pathophysiology

of Human Reproduction, University of Cagliari,

Cagliari, Italy

G. Carri

Department of Obstetrics and Gynecology,

Università Cattolica del Sacro Cuore,

Roma, Italy than white women. They are almost always benign and frequently asymptomatic. When symptomatic, however, uterine leiomyomas may cause heavy menstrual bleeding, pelvic pressure or pain, dyspaurenia, urinary frequency, incontinence, and urgency or constipation. Women with submucosal fibroids may also experience reproductive problems such as infertility, miscarriages, premature labor, or peripartum hemorrhage. After myomectomy, successful pregnancy rates occur in $53 \%$ to $70 \%$ for submucous fibroids and $58 \%$ to $65 \%$ for intramural and subserosal fibroids $[1,2]$.

The mechanisms by which myomas adversely affect fertility are several: displacement of the cervix which may reduce exposure to sperm; enlargement or deformity of the uterine cavity which may interfere with sperm migration and transport; obstruction of the proximal fallopian tube; altered tubo-ovarian anatomy, interfering with ovum capture; increased or disordered uterine contractility that may hinder sperm or embryo transfer or nidation; distortion or disruption of the endometrium; and impaired endometrial blood flow [3].

The removal of leiomyomas with preservation of the uterus is indicated in symptomatic women who wish to preserve their reproductive function and anatomic integrity. Once the decision is made to proceed with the myomectomy, the surgeon must decide whether the patient would be better suited to the laparoscopic or the laparotomic approach. This decision depends on several factors, including the size, the number, the location of the fibroids on the uterus, and the experience of the surgeon. The latter is perhaps the most important determinant as the completeness of the myomectomy and the adequacy of the reconstruction of the uterus after myomectomy will determine the subsequent function of the organ in relieving the symptoms and restoring reproductive potential.

Some investigators have found that uterine myoma have no effect on in vitro fertilization (IVF) outcomes unless they 
distort or displace the uterine cavity; other investigators suggest that IVF success rates are also lower in women with intramural myomas, particularly when larger than $5 \mathrm{~cm}$ in diameter. Recent findings suggest that the size of the fibroids is positively related to implantation failure, especially when the diameter of the lesion exceeds $4 \mathrm{~cm} \mathrm{[4]} \mathrm{and} 5 \mathrm{~cm} \mathrm{[5].}$

The size, the number, and the location of fibroids that can be removed by a minimally invasive approach depend largely on the endoscopic skills and experience of the surgeons [6]. The latest advancements in terms of technical expertise and surgical instrumentation have allowed minimal-access surgery to become even more minimal with the development of single-access laparoscopic surgery (SALS). Reducing the number of working ports decreases the risk of bleeding, infection, concomitant iatrogenic visceral injury, hernia formation with better pain control, and cosmetic outcome. After initial applications on animal models, the subsequent development of specialized purpose-built instrumentation coupled with advances in skills led to the successful application of single-port access laparoscopy (SPAL) in human patients and this minilaparoscopic procedure has emerged as one of the viable, feasible, and widely applicable minimally invasive procedures. Preliminary advances in SPAL were applied to urologic and gastrointestinal surgery, and only recently papers have been published about the application of SPAL also in gynaecology $[7,8]$. These preliminary experiences have shown good results in terms of feasibility, operative time, and complications particularly in the treatment of adnexal pathologies. At the moment, few experiences on SPAL myomectomy have been reported [8]. We present our technique to perform SPAL myomectomy using a new re-usable trocar (X-Cone).

\section{Method}

A 38-year-old infertile woman affected by bilateral sactosalpinx and uterine myoma underwent myomectomy and bilateral salpingectomy by single-access transumbilical laparoscopy.

The patient had previously undergone appendicectomy surgery. She had a regular menstrual cycle with a normal hormonal profile. Abdomino-pelvic examination was negative while transvaginal ultrasound found an anterior intramural myoma of $4 \mathrm{~cm}$ in diameter which distorted the endometrium and bilateral hydrosalpinx.

Laparoscopy was performed using a reusable single-site trocar with five integrated access ports (S-Portal X-Cone, Karl Storz, Tuttlingen, Germany). This new device consists of two symmetrical metal half-shells that are connected and sealed by one large silicon cap containing the access port. Once in the peritoneal cavity, the two half-shells fit together to form an $\mathrm{X}$ shape and the final access portal. The silicon cap comprising three $5-\mathrm{mm}$ and one $12-\mathrm{mm}$ port is connected on top of the X-Cone, which forms an airtight seal. A clamp integrated into the rubber sealing prevents the $\mathrm{X}$-Cone from opening unintentionally. The external and internal diameters at the level of the X-Cone's waistline are 25 and $20 \mathrm{~mm}$, respectively. Under general anesthesia, each patient was positioned in the dorsal lithotomic position with both legs supported in Allen stirrups and the arms resting alongside the body. An intrauterine device was used to mobilise the uterus. A $2.0-\mathrm{cm}$ intraumbilical vertical skin incision and a $2-2.5-\mathrm{cm}$ rectus fasciotomy were performed to enter the peritoneal cavity. The single-port trocar was inserted into the abdominal cavity and the abdomen was insufflated to $12 \mathrm{mmHg}$. The patient was then put in a Trendelemburg position. Following placement of the $\mathrm{X}$-Cone, the surgeon stood behind the patient's left shoulder at the level of the patient's right shoulder, and the monitor was positioned between the patient's legs. We routinely used a rigid $30^{\circ}, 5$-mm-diameter, 50 -cm-length Hopkins high definition laparoscope in combination with a high definition three-chip video camera (Karl Storz) [8, 9].

For the bilateral salpingectomy, rigid single curved forceps or scissors (S Portal, Karl Storz) and a standard straight bipolar dissector or device with grasping, coagulating, and transecting functions (En Seal; Ethicon) were used simultaneously. A $10-\mathrm{mm}$ endobag through the $12-\mathrm{mm}$ X-Cone access was used to remove the specimens.

The myometrium overlying the fibroid is injected with a 1:20 diluted solution of vasopressin using a laparoscopic needle. After the injection of 40 to $60 \mathrm{~mL}$ of solution, the uterus pales, indicating diminished blood flow. The myometrial incision was made with a flexible monopolar hook (Karl Storz) and was deep enough to the dissection plane between the myoma and the myometrium. Enucleation of the myoma was performed with a 10-mm myoma drill (Fig. 1).

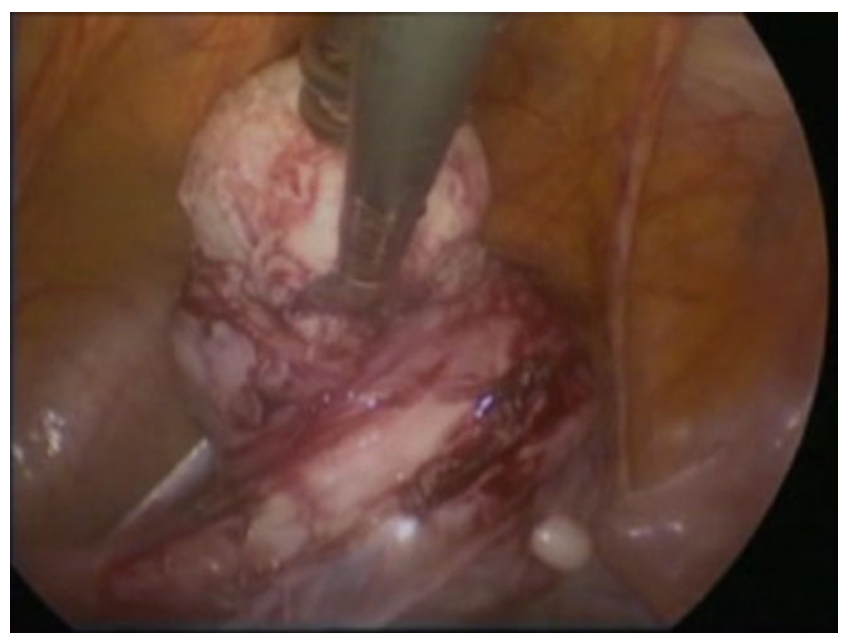

Fig. 1 Enucleation of the myoma performed with a 10-mm myoma drill 
An intracorporeal double-layer suture of myometrium was performed by 2-0 absorbable barbed monofilament (VLoc 180, Covidien). For stitching, a straight needleholder and a curved Kelly forcep as a contra-needleholder were used. Removal of the specimen was obtained under a vision with a 10-mm morcellator (Rotocut, Karl Storz) through the $\mathrm{X}$-Cone $12 \mathrm{~mm}$ port.

No intraoperative and postoperative complications were detected. The estimated blood loss was less than $20 \mathrm{ml}$ and the total operative time (skin to skin) was $94 \mathrm{~min}$.

The patient was discharged after 1 day of hospitalization with a complete satisfaction in terms of pain and cosmetic outcome. The postoperative control after 1 month revealed a complete recovery of the umbilical scar at clinical exploration and of the uterus at ultrasound.

\section{Findings - discussion}

We describe a new surgical approach for transumbilical single-port access surgery in infertile patient with uterine leiomyoma and bilateral sactosalpinx. The surgical strategy planned for this patient has been bilateral salpingectomy and myomectomy to improve the IVF pregnancy rate. In our experience, we believe that the removal of the myoma in infertile patients which are $4 \mathrm{~cm}$ or more in diameter, which distort the endometrium causing defects in embryo implantation, is effective. SALS provides an opportunity to further enhance the cosmetic benefits of minimally invasive surgery while minimising the potential morbidity associated with multiple incisions but it has to be taken in consideration that SPAL approach has intrinsic limits such as absence of triangulation, inline vision, and instruments crowding. Therefore, to improve the performance and the application of this surgery, some suggestions and techniques should be taken into account. To facilitate the correct ergonomy, the monitor should be placed between the patient's legs and the first surgeon should stand by the left shoulder (lateral to the head of the patient) to recreate the correct axis for eyes-hands-monitor; the assistant should stand at the level of the patient's right shoulder. Since a third assistant's hand is not required in single-port procedures, an intrauterine device should be placed to aid in the manipulation of the uterus and exposition of the surgical field. The use of a $5-\mathrm{mm}, 30^{\circ}$ telescope is mandatory for both avoiding inline view and a constant clashing between instruments and a video camera and a $50-\mathrm{cm}$ extendedlength scope is useful since it allows the assistant to move the scope without hindering the surgeon's movements outside of the abdominal cavity.

During laparoscopic surgery, instrument triangulation allows proper tissue retraction which is essential for proper dissection along anatomical tissue planes and helps to avoid crossing between the instruments and the camera. The use of at least one flexible or curved instrument moves the shaft's axis out of the center sufficiently, thus allowing the surgeon to operate with some degree of triangulation. The use of curved instruments (single or double curve) consents the mobilization and traction of the organs using rotating, forward, and backward movements.

To reduce crowding instruments, the profile should be as slim as possible. If one camera and two instruments occupy the multichannel port, varying lengths prevent the bulkiest portion of each instrument (the external handle) from overlapping extracorporeally. A straight rotating handle is preferable.

In addition, multifunctional devices capable of grasping, dissecting, coagulating, and cutting overcome the limitation imposed by the reduced numbers of ports.

Stitching and suturing remain one of the most difficult skills in laparoscopy and more so in the single-port approach [9-12]. Standard rules have to be followed in order to simplify the technique as much as possible. To overcome the difficulty of the intracorporeal knot, we decided to use a barbed monofilament capable of safely fixing the tissue with a constant tension along the wound. A double-layer suture was needed to correctly close the myometrium, and to reduce adhesions an intraflexion suture at the level of the perimetrium was performed. Continuous suturing technique and absence of tying knots permit a considerable reduction in operating time. A good rule to keep in mind in performing laparoscopic stitching is that the suture axe should be parallel to the needleholder and perpendicular to the needle axe and in the single-port approach this means that the uterine incision, at the level of the anterior or posterior wall, should always be longitudinal. Even if the cosmetic advantage of the SPAL is realistic and more patients start to request this particular approach, it is important to define correct indications and to standardize techniques. With this report, we describe the technique used to perform SPAL myomectomy but we will need further experience and studies to correctly define the best reproducible technique.

\section{Conclusion}

Single-port laparoscopic myomectomy with X-Cone is feasible; however, further reports are needed to better define patients selection, to standardize the technique, and to develop specific instruments.

Declaration of interest The authors report no conflicts of interest. The authors alone are responsible for the content and writing of the paper. 


\section{References}

1. Luciano AA (2009) Myomectomy. Clin Obstet Gynecol 52 (3):362-371

2. Baird DD, Dunson DB, Hill MC et al (2003) High cumulative incidence of uterine leiomyoma in black and white woman. Am J Obstet Gynecol 188:100-107

3. (2008) The Practice Committee of the American Society for Reproductive Medicine in collaboration with the Society of Reproductive Surgeons. Myomas and reproductive function. Fertil Steril 90(Suppl 3): S125-130

4. Oliveira FG, Abdelmassih VG, Diamond MP, Dozortsev D, Melo NR, Abdelmassih R (2004) Impact of subserosal and intramural uterine fibroids that do not distort the endometrial cavity on the outcome of in vitro fertilization-intracytoplasmic sperm injection. Fertil Steril 81(3):582-587

5. Bulletti C, DE Ziegler D, Levi Setti P, Cicinelli E, Polli V, Stefanetti M (2004) Myomas, pregnancy outcome, and in vitro fertilization. Ann NY Acad Sci 1034:84-92
6. William H, Parker MD (2007) Uterine myomas: management. Fertil Steril 88(2):255-271

7. Kim T-J, Lee Y-Y, Kim KMJ, CJ KH, Choi CH, Lee J-W, Kim B-G, Bae D-S (2009) Single port access laparoscopic adnexal surgery. J Minim Invasive Gynecol 16:612-615

8. Lee JH, Choi JS, Jeon SW, Son CE, Lee SJ, Lee YS (2010) Eur J Obstet Gynecol Reprod Biol 153:81-84

9. Kaouk JH, Haber GP, Goel RK, Desai MM, Aron M, Rackley RR et al (2008) Single port laparoscopic surgery in urology: initial experience. Urology 71(1):3-6

10. Fader AN, Escobar PF (2009) Laparoendoscopic single-site surgery (LESS) in gynecology oncology: technique and initial report. Gynecol Oncol 114(2):157-161

11. Mereu L, Angioni S, Melis GB, Mencaglia L (2010) Single access laparoscopy for adnexal pathologies using a novel reusable port and curved instruments. Int J Gynecol Obstet 109(1):78-80

12. Fagotti A, Fanfani F, Marocco F, Rossito C, Gallotta V, Scambia G (2009) Laparoendoscopic single-site surgery (LESS) for ovarian cyst nucleation: report of first 3 cases. Fertil Steril 92(3):1168: $13-16$ 